\title{
A Fast Continuous Magnetic Field Measurement System Based on Digital Signal Processors
}

\author{
G.V. Velev, R. Carcagno, J. DiMarco, S. Kotelnikov, M. Lamm, A. Makulski, V. Maroussov, \\ R. Nehring, J. Nogiec, D. Orris, O. Poukhov, F. Prakoshyn, P. Schlabach, J.C. Tompkins
}

\begin{abstract}
In order to study dynamic effects in accelerator magnets, such as the decay of the magnetic field during the dwell at injection and the rapid so-called "snapback" during the first few seconds of the resumption of the energy ramp, a fast continuous harmonics measurement system was required. A new magnetic field measurement system, based on the use of digital signal processors (DSP) and Analog to Digital (A/D) converters, was developed and prototyped at Fermilab. This system uses Pentek 610216 bit A/D converters and the Pentek 4288 DSP board with the SHARC ADSP-2106 family digital signal processor. It was designed to acquire multiple channels of data with a wide dynamic range of input signals, which are typically generated by a rotating coil probe. Data acquisition is performed under a RTOS, whereas processing and visualization are performed under a host computer. Firmware code was developed for the DSP to perform fast continuous readout of the A/D FIFO memory and integration over specified intervals, synchronized to the probe's rotation in the magnetic field. C, $\mathrm{C}++$ and Java code was written to control the data acquisition devices and to process a continuous stream of data. The paper summarizes the characteristics of the system and presents the results of initial tests and measurements.
\end{abstract}

Index Terms-Digital signal processors, Magnet field effects, Magnetic field measurements

\section{INTRODUCTION}

$\mathrm{F}$ IELD errors from superconducting magnet persistent currents have a significant role in the operation of modern accelerators due to their large amplitude and strong time dependence [1]-[2]. These effects, first observed in Tevatron chromaticity variations (up to 70 units) during the dwell at injection [2] were a consequence of the field sextupole decay. These variations were unacceptable and corresponding corrections were developed [3] and have been recently revised for the optimization of Run II operation [4].

It was observed that some of the dynamic changes in the magnetic field occur for relatively short periods of time. A typical example is the fast field change, dubbed a "snapback",

Manuscript received September 19, 2005. This work was supported by the U.S. Department of Energy.

G. V. Velev, R. Carcagno, J. DiMarco, S. Kotelnikov, M. Lamm, A. Makulski, J. Nogiec, D. Orris, P. Schlabach,, J.C. Tompkins are with Fermilab, Batavia, IL 60510 USA (phone: 630-840-2203; fax: 630-840-2383 e-mail: velev@fnal.gov).

V. Maroussov is with Purdue University, West Lafayette, IN 47907 USA.

O. Poukhov and F. Prokoshyn are with JINR, Joliot-Curie 6, Dubna, Russia. which occurs during the first few seconds of the resumption of the energy ramp. The duration of the snapback is of the order of several seconds (typical duration for the Tevatron injection of $30 \mathrm{~min}$ is $\sim 8 \mathrm{~s}$ ) and it strongly depends on magnet history. An accurate correction is needed for this short period in the accelerator cycle if beam losses are to be minimized.

Recently discovered, relatively large sextupole decay $\sim 0.25$ units from the main field - after short duration ( 6-20 s) injections added additional complexity to our understanding of the dynamic processes in superconducting magnets [5]. A newly proposed scaling law [7], states that the field decay amplitude during beam injection and the current necessary to resolve it (this current is usually a function of snapback time) are strongly correlated for magnets of similar design. To confirm this statement, especially in the regime of very short injection plateaus, where the decay amplitudes are close to zero, additional measurements are needed.

To meet the requirement for these measurements, including future special operational needs for the Tevatron and the LHC, a fast rotating coil magnetic measurement system which can accurately and synchronously sample all the required field harmonics was considered. This type of system based on a digital signal processor and A/D converters was developed, prototyped and built at Fermilab and is described in this paper. A comparison with a previous Fermilab system, based on digital integration modules (PDI's) will also be presented. Finally, the first results from measurements performed on the Tevatron dipoles with this new system are summarized, including new results on the scaling law and sextupole decays after short duration injections.

\section{Description OF THE MEASUREMENT SYSTEM BASED ON A Digital SignAl PROCESSOR}

The block diagram of the DSP measurement system is shown in Fig. 1. The three major components are outlined in separated boxes (from left to right): the Signal Conditioning Board (SCB), the Programmable Gain Amplifiers Module (PGA) and the Signal Processing Boards (SPB). In addition, a harmonic trigger module (HTM) and an encoder emulator for performing offline tests were developed internally and utilized with the system. All the modules are VME standard except the SCB.

\section{A. Signal Conditioning Board}

The SCB was designed and produced at Fermilab. The 


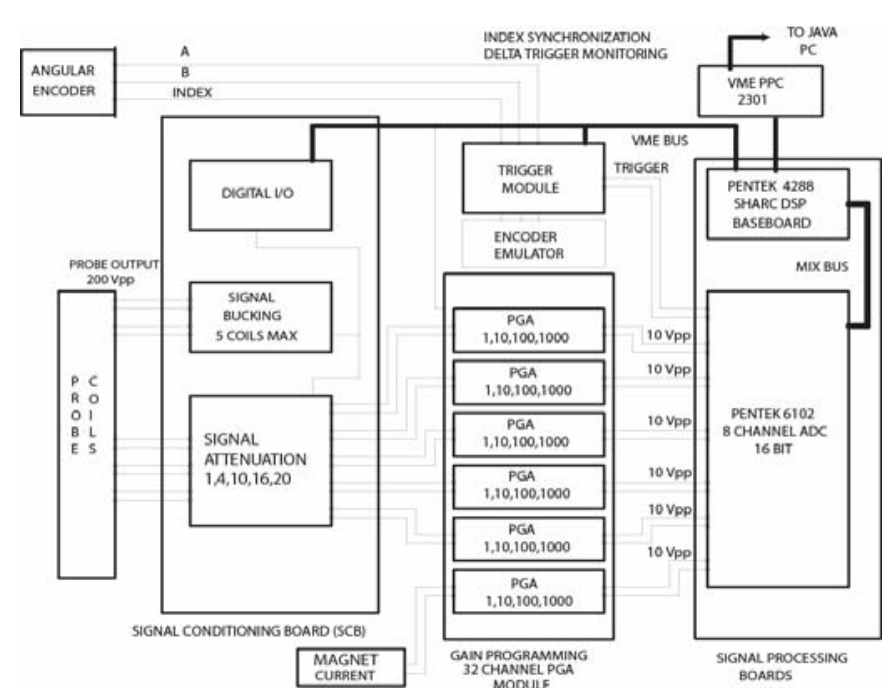

Fig. 1. Schematic diagram of the measurement system. The three major blocks of the system signal manipulation (Signal Conditioning Board, Programmable Gain Amplifier and Signal Processing Boards) are outlined in separated boxes.

SCB accepts input signals from up to 10 sources (coils); in the current version of the board only 5 inputs are activated. The maximum input amplitude is $200 \mathrm{Vpp}$, which gives us the capability to utilize large integral rotating coil probes, similar to a $7 \mathrm{~m}$ long probe used in production measurements of the LHC interaction region quadrupoles. Voltages from up to 5 coils can be bucked together. A current summing technique has been used instead of the typical voltage summing method for analog bucking, allowing simultaneous recording of raw and bucked signals. To obtain small offsets and offset drifts, low thermal EMF Coto relays (model 3602) have been used for the control of the attenuation section. The SCB provides attenuations of 1, 4, 10, 16, 20 for each signal except the bucking one.

\section{B. Programmable Gain Amplifiers Module}

The PGA module is based on the commercially available VMIVME-3419 board. It is a 32 channel signal conditioning board with a range of manual gain selections: $1,10,100$ and 1000 ; and full scale ranges from $\pm 10 \mathrm{mV}$ to $\pm 10 \mathrm{~V}$. Threepole, active, filters are used on the input of every channel. In our case the filters were selected for a cut-off frequency of 1 $\mathrm{kHz}$. The major characteristics of the board are summarized elsewhere [8].

The second part of the PGA block is an in-house made Programmable Gain Control Mezzanine Card (PGCMC). It is designed to provide the user with the ability to change gains automatically using a 12-bit data word from a standard TTL digital I/O card. In our case, we use the VMIVME-2510B digital I/O card. This gives us the ability to change the amplifier gains "on the fly" without powering down the VME crate, changing the jumper settings for each desired channel. This feature is important for software realization of automatic gain control of the measurement system.

\section{Signal Processing Boards Block}

Two boards form the SPB block. The first one is a Pentek
A/D -D/A converter, model 6102 [9]. It is a high performance, 8-channel converter for data acquisition and has differential inputs to the $\mathrm{A} / \mathrm{D}$ section featuring 16-bit resolution, a maximum sampling frequency of $250 \mathrm{kHz}$ and 16k samples FIFO per channel. This board is attached to a second one, the Pentek model 4288 [10], which is the central element of the system. This board contains the $40 \mathrm{MHz}$ AD2106 Super Harward Architecture Computer (SHARC) DSP by Analog Devices. The SHARC processor delivers up to 120 MFLOPS of computing power. Both boards communicate through a proprietary high speed mezzanine bus, Intel's Modular Interface eXtension (MIX) [11].

The conditioned signals are fed to the input of the A/D converter and sampled at $40-50 \mathrm{kHz}$. When the "FIFO HALF-FULL" is enabled, the digital buffer is transferred to the local SRAM, accessible directly from the SHARC processor. More details about data processing are presented in section III, which describes the DSP firmware code.

\section{Harmonic Trigger Module}

The HTM board was developed and fabricated at Fermilab. The main purpose of this board is to condition the signals coming from the angular encoder which usually is directly connected to the rotating probe shaft. It accepts the three encoder signals, named $0^{\circ}, 90^{\circ}$, and zero position or index pulse, divides (by 1, 2, 4, 8 or 16) or multiplies (1, 2 or 4) a selected encoder signal and provides it to the DSP for a proper voltage-to-flux integration. In addition, divisions of 8 and 16 of the encoder pulses can be provided as an independent trigger signal to a Digital Voltmeter (DVM) in the case that such a device is used for additional monitoring.

Different features and checks were implemented into the HTM board. It can enable and disable the trigger signals from control sequences coming either on the VME bus or from the front panel. An internal counter can be initialized to capture the time difference between two index pulses and compare it with the expected value. This information is provided to the VME bus and can be used for monitoring purposes.

\section{DSP CODE}

The DSP firmware code was designed and written in-house. The major task for this code is to transfer on-line the information from A/D converter FIFO buffers into the processor memory, integrate the input voltages to the fluxes and transfer the flux values to the VME accessible memory for reading by the control VME PPC computer. Special treatment of the encoder pulses and the current reading channel is performed.

A block diagram of the firmware code is shown in Fig.2. The $16 \mathrm{k}$ word A/D FIFO is read in DMA mode into two $4 \mathrm{k}$ word alternating input buffers. They are the major holders of the data before signal processing. When enabled, the specific A/D channel "FIFO HALF-FULL" interrupt triggers FIFO readout. In standard operation, the first $\mathrm{A} / \mathrm{D}$ channel contains the digitally conditioned signal from the angular encoder of the system (see Fig.1). When this signal is digitized, an 


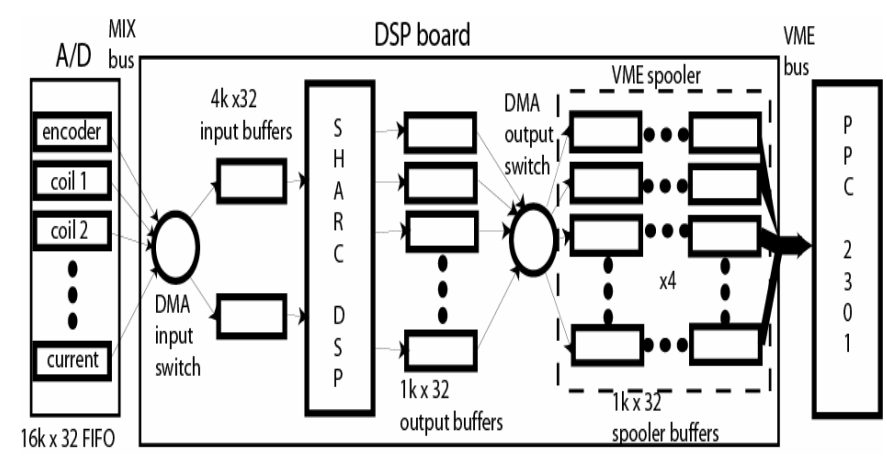

Fig. 2. DSP data processing and firmware code diagram

integration mark to the leading edge of each encoder trigger pulse are determined and stored in one of the $1 \mathrm{k}$ sample output buffers (the number of the output buffers is equal to the number of $\mathrm{A} / \mathrm{D}$ channels) in the DSP memory. When the output buffer is full, a program-med interrupt is invoked which starts the DMA transfer of the information to the external DSP VME memory (VME spooler, 512k words). For every D/A channel, there are four buffers in the VME spooler, which keep the data and service/control information as well. These buffers are accessible from the control PPC computer through the VME bus.

The conditioned analog signals from the probe are read and digitized like the encoder signals. After the digitized information is transferred to the DSP input buffers, an integration procedure is started. The magnetic fluxes are obtained between the integration marks and they are written into the output DSP buffers. The integration marks, corresponding to the encoder index (zero) pulses, are used to reset the integration counters. Utilizing the same DMA procedure as described for the encoder channel, the data are transferred to the VME spooler.

In case of the current reading channel, a special integration over the power cycle (in our case $60 \mathrm{~Hz}$ ) is needed. The firmware code performs this integration and synchronizes the current output with the magnet flux output with a time resolution less than $1 \mathrm{~ms}$ in case of a $1 \mathrm{kHz}$ encoder frequency. This synchronization is much better than in our PDI type system where the current is digitized with an external DVM.

Specialized C and C++ code was written for the VME PPC 2301 computer (Fig.1) running VxWorks, which communicates directly, in real time, with the DSP SHARC. The main task for this computer is to sequentially read the spooler buffers through the VME bus and to stream the data to the Java on-line analysis code running on the workstation [12]. The Java code responsible for analysis, data archival, visualization, and measurement control is based on the EMS framework [13]. In response to requests received from the Java code, the VME processor controls the attenuations in the SCB, gains in the PGA, and signal conditioning of the HTM and initializes all the modules, including the DSP and the A/D converters.
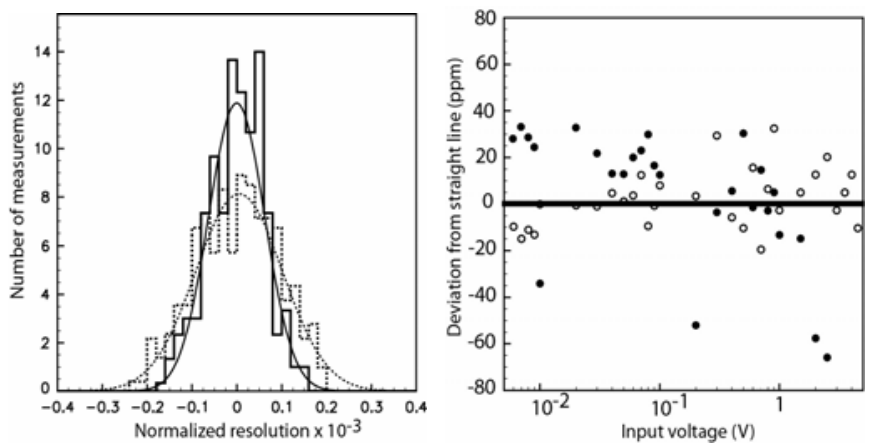

Fig. 3. Comparison of the normalized resolution (left), explained in the text, measured for the DSP system (solid line) and for PDI type system (dashed line). The linearity of the DSP system (open points) and the PDI type system (filled points) is shown in the right plot.

\section{COMPARISON BETWEEN DSP AND PDI TYPE MEASUREMENT SYSTEMS}

The major design specifications of the system can be summarized as: to have the ability to accept and manipulate input signals with large dynamical range, to take data continuously with a high input rate for a long interval of time (a test was performed with 2 days duration at $5 \mathrm{~Hz}$ simulated probe rotation) and to have at least the same or better resolution and linearity than the PDI type measurement system.

Several tests were performed to check these requirements. We compared our DSP measurement system with another Fermilab built system using the same Java EMS framework [14], but based on PDI VME modules (model 5035) made by Metrolab [12]-[15]. This system did not have the ability to work in a continuous mode because of limitations in the PDI module readout; the maximum achieved effective probe rotation speed corresponded to $1.5-1.8 \mathrm{~Hz}$ with 64 encoder pulses per revolution.

The next step was to compare the resolution and linearity of the systems. Using a calibrated voltage source, $1 \mathrm{~V}$ was supplied to the input of the one of the system channels. The gain and attenuation for this test channel were set to one. In Fig 3, the left hand plot shows the distributions of a normalized resolution, $\left(\mathrm{x}^{\mathrm{i}}-\mathrm{x}^{\text {mean }}\right) / \mathrm{x}^{\text {mean }}$, for 500 independent measurements, where $\mathrm{x}^{\mathrm{i}}$ is the result from the $\mathrm{i}^{\text {th }}$ measurement. In this test, the DSP system achieves 1.5 times better resolution than the PDI setup.

The right-hand plot in Fig.3 shows the linearity deviation from a straight line crossing zero in ppm of the input range. The maximal observed deviation is $\sim 30 \mathrm{ppm}$ (open points) for the DSP system which is better than the maximal measured PDI (filled points) linearity deviation of $\sim 70 \mathrm{ppm}$.

\section{Results on the TeVatron Dipoles}

The DSP system was utilized to perform measurements on Tevatron dipoles on a test stand in the Fermilab Magnet Test Facility. All the magnets had been quenched and prepared with the same pre-cycle ( 60 min flat-top at $980 \mathrm{GeV}$, followed by 5 min back-porch at $150 \mathrm{GeV}$, fast reset at $90 \mathrm{GeV}$ and a 


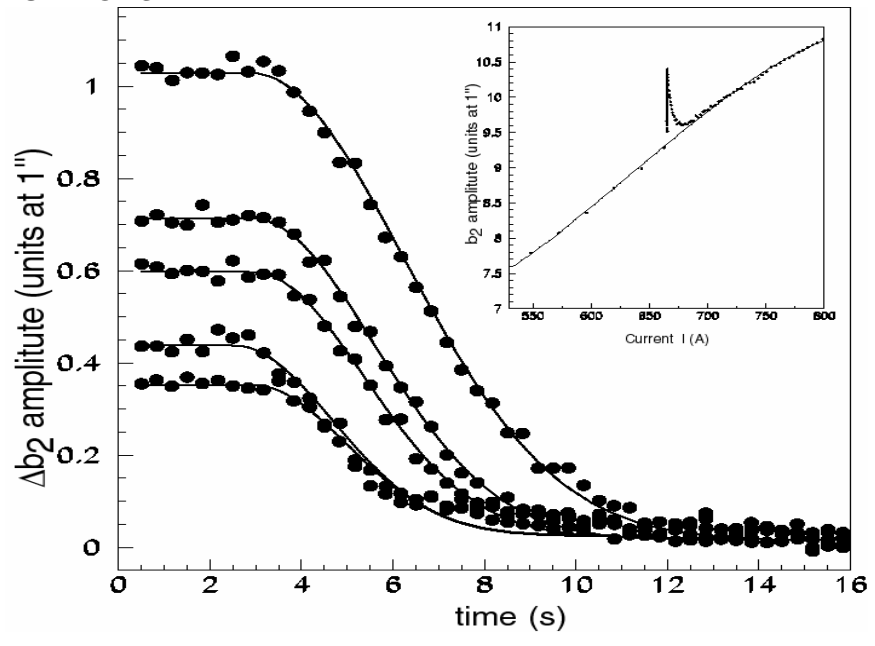

Fig. 4. Typical sextupole decay amplitudes $\Delta \mathrm{b}_{2}$ versus the snapback time for the Tevatron dipole TB0834. The dots and the fitted curves correspond, from bottom to top, $6 \mathrm{~s}, 60 \mathrm{~s}, 5 \mathrm{~min}, 10 \mathrm{~min}$ and 30 min durations of the injection porch. The insert shows how the correction for the sextupole hysteresis loop was done using the polynomial of third order.

ramp to $150 \mathrm{GeV}$ injection porch) which are as close as possible to the actual Tevatron cycle [4]. After the pre-cycle, we varied the duration of the injection porch $(6,12,20,30$, 60 s and $1,5,10,15,20,25,30 \mathrm{~min})$ measuring the sextupole field decay and snapback. Typical $b_{2}$ snapbacks for different injection porch durations are shown in Fig.4. To decouple the snapback from the underlying hysteresis loop, we parametrized $b_{2}$ with a third order polynomial in the regions $0.54-0.65 \mathrm{kA}$ and $0.70-0.80 \mathrm{kA}$, interpolated its value to the injection plateau at $0.66 \mathrm{kA}$, and subtracted it from the sextupole loop (see the insert in Fig.4). After a 6s injection duration, the sextupole field in the magnet (TB0834) decays 0.35 units which is unexpectly large compared to the predictions of the existing models and empirical parametrizations. The other two measured magnets (TC0710, TC1077) showed similar behavior. This is displayed in Fig.5 where all the points from short injection porches populate the region of $\Delta \mathrm{b}_{2}$ between 0.2-0.4 units.

The performed measurement gave us the ability to check the scaling law proposed in [7]. From Fig.5 one can conclude that the three magnets have different slopes of $0.185,0.201$ and 0.217 and $1 / \mathrm{A}$ with average statistical error of 0.002 (in magnet TC0710 an intercept statistically inconsistent with 0 units is observed). This preliminary result contradicts the assumption made in [7] that all magnets with given design should have the same linear dependence.

\section{CONCLUSIONS}

A new magnetic measurement system was developed, prototyped and built at Fermilab. The system is based on A/D converters and a Digital Signal Processor with the ability to perform large dynamic range signal conditioning. It outperforms the existing PDI type systems in its ability to take continuous data at high rate. The resolution and linearity tests showed that the DSP system is $1.5-2$ times better than the previous utilized system.

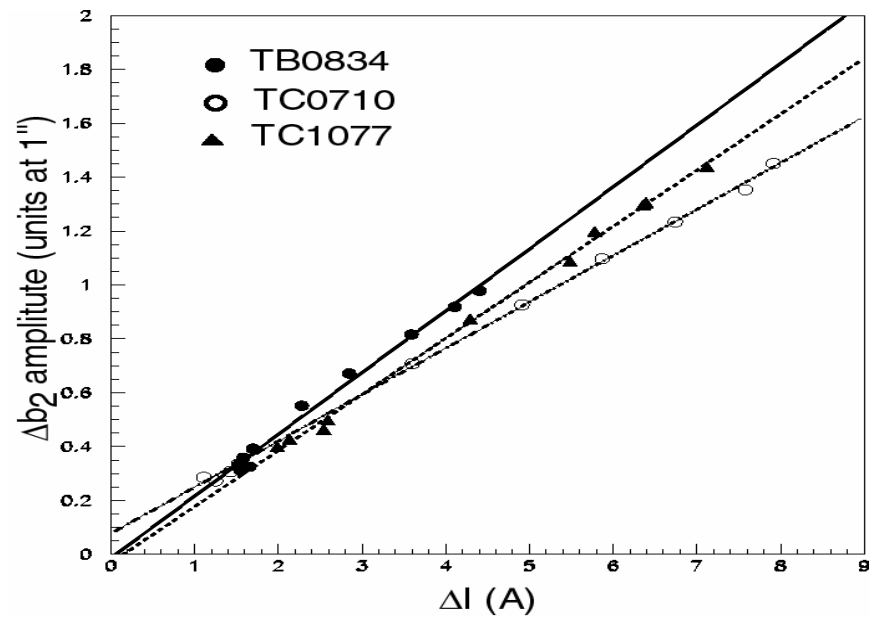

Fig. 5. The sextupole decay amplitude $\Delta \mathrm{b}_{2}$ versus the snapback current $\Delta \mathrm{I}_{\text {snbck }} \sim \Delta \mathrm{B}_{\text {snbck }}$ for three Tevatron dipoles (TB0834, TC0710, TC1077). The linear parametrization is also shown. The measurements are performed with DSP system.

Using this new DSP-based system, magnetic measurements were performed on three Tevatron dipoles. The existence of the relatively large decay in the magnets after very short injection porches $(6,12,20 \mathrm{~s})$, first reported in [5], was confirmed. In addition, an accurate check of the scaling law, proposed in [7], was performed. The Tevatron dipoles show close but distinguishably different slopes in the dependence of $\Delta \mathrm{b}_{2}$ on $\Delta \mathrm{I}_{\text {snbck }}$.

A second system based on the same hardware with an optimized DSP firmware will be built in the near future.

\section{REFERENCES}

[1] D. A. Finley et al., "Time dependent chromaticity changes in the Tevatron", in Proc. 12 $2^{\text {th }}$ Part. Acc. Conference, Washington, DC, 1987.

[2] H. Brueck et al., "Time Dependent Field Distortions from Magnetization Currents in the Superconducting HERA Magnets”, Cryogenics, 30, pp. 605-609, 1990

[3] D.E. Johnson and D.A. Herrup "Compensation of time Varying Field in the Tevatron Superconducting Magnets", in Proc. $12^{\text {th }}$ Part. Acc. Conference, 1989.

[4] G. V. Velev el al., "Measurements of Sextupole Decay and Snapback in Tevatron Dipole Magnets", in Proc. $9^{\text {th }}$ EPAC, Lucerne, 2004.

[5] G. V. Velev el al., "Measurements of Field Decay and Snapback Effect on Tevatron Dipole and Quadrupole Magnets", in Proc. $21^{\text {st }}$ PAC, Knoxville, TN, 2005.

[6] G. Anala et al., "Advances in the Understanding and Operations of Superconducting Colliders", in Proc. 21 ${ }^{\text {st }}$ PAC, Knoxville, TN, 2005.

[7] G. Ambrosio et al.," A Scaling Law for Predicting Snapback in Superconducting Accelerator Magnets”, IEEE Trans. of Applied Superconductivity, Vol. 15, No. 2, June 2005, pp. 1217-1220.

[8] VMIVME-3419, 32 Channel Signal Conditioning Board with Selectable Gain and Built-in-Test, VMIC, Doc. No. 500-003419-000C, May 12, 1997.

[9] Operating Manual, Model 6102, Pentek, Inc., September 28, 1998.

[10] Operating Manual, Model 4288, Pentek, Inc., March 27, 2000.

[11] The MIX Interface: A Mezzanine Bus for VME,. Available: http://www.pentek.com/Tutorials/MIX/MIX.htm.

[12] G. V. Velev at al., "Field Quality of the LHC Inner Triplet Quadrupoles Being Fabricated at Fermilab", in Proc. $20^{\text {th }}$ PAC, Portland, OR, 2003.

[13] J.M. Nogiec et al. "EMS: A Framework for Data Acquisition and Analysis," in Proc. ACAT, Batavia, IL, 2000.

[14] J.M. Nogiec et al., "A Flexible and Configurable System to Test Accelerator Magnets," in Proc. 19 ${ }^{\text {th }}$ PAC, Chicago, IL, 2001.

[15] PDI 5035 Precision Digital Integrator, METROLAB Instruments SA, June 1994. 